\title{
The Vital Role of Pharmacy Benefit Management Firms in Health Services Research
}

\begin{abstract}
At the AMCP annual meeting in New Orleans in May 1997, a panel discussion entitled, "Horizons for Health Services Research in PBMs: An Emergent Public Health Mandate," addressed the need for solid database information in health care research. Among the most promising sources for valid data: pharmacy benefit management firms.
\end{abstract}

I n 1989, Henry R. Manasse, former dean of the University of Illinois Col-lege of Pharmacy, detailed the problems of "drug misadventuring" as an issue of national public policy. ${ }^{1}$ He described drug misadventuring as a broad array of phenomena associated with negative drug experience whether resulting from action or inaction by physicians, pharmacists, patients, manufacturers, policymakers, or third party payors. Later, University of Arizona College of Pharmacy Dean Lyle Bootman and his colleague Jeffrey Johnson calculated the economic cost of drug misadventuring in American society at about $\$ 76.6$ billion in unnecessary health care expenditures. ${ }^{2}$ The human toll and economic burden from inappropriate drug use within our health care system, the growing influence of economic considerations in the use of pharmaceuticals, and employer demands for provider accountability all were part of the impetus behind the formation of pharmacy benefit management (PBM) firms. Drug misadventuring has become a major public health problem. Consequently, it needs to become an issue of public debate and policy correction for enhancement of patient outcomes and cost containment.

\section{POTENTIAL FOR SOLUTIONS TO DRUG MISADVENTURING}

From the 1940s to the Nixon-Ellwood HMO strategy (1969-1973), there had been a long tradition of social science research into the kind of services delivered within the prepaid group practice movement. ${ }^{3}$ Since PBMs have become vast storehouses of data on pharmacotherapy, monumental opportunities exist through applied research to resolve much of the societal drug misadventuring and then to find out what really works best in pharmacotherapy from an evidence-based approach. ${ }^{4}$ The word mandate from the session title can mean a request or an appeal or, alternatively, a decree or an order. The spirit of the session's presenters was to appeal to volunteerism within the private PBM industry for conducting health services research before private or governmental regulators and payors issue decrees or orders in some direction.

PBMs hold the possibility for an initial foray into performing populationbased outcomes research in the managed care era. ${ }^{5}$ Because pharmacotherapy is critical to all health care outcomes, these pharmacy-based information storehouses may contain potential solutions to much of the societal drug misadventuring that has been documented over the years, beyond merely the cost savings of improving such interventions. However, PBM data remain proprietary. There has not been a noticeable initiative in engaging outside academic research assistance for analyses nor a trend of studies conducted in-house and 
published in the accepted peer-reviewed scientific literature.

In his keynote address at the AMCP meeting in Orlando, David Satcher, M.D., head of the Centers for Disease Control and Prevention (CDC), summoned pharmacy practitioners to work with medicine and public health to meet new challenges in American society through population-based prevention. ${ }^{6}$ Much remains to be done in delineating the multifactored causes of modern morbidity and mortality as well as in identifying risk factors-both of which are integral to launching social prevention strategies. ${ }^{7}$ Dr. Satcher invited leaders of AMCP to work with CDC officials to educate providers, patients, and the public on issues of critical public health importance.

In the spirit of Dr. Satcher's address, with this article the authors seek to foment deeper discussions among PBM staffs, the pharmaceutical industry, academia, managed care organizations (MCOs), and policymakers toward a planned research agenda on pharmacotherapy's contributions to public health outcomes.

\section{HEALTH SERVICES RESEARCH POTENTIAL}

In 1995, the Group Health Association of America and the U.S. Agency for Health Care Policy and Research jointly sponsored a conference on building bridges between the HMO and the health services research communities. ${ }^{8}$ The basis for such an endeavor was that HMOs had data and could easily gather more for conducting health services research on the quality and cost of care provided. The other advantage included information on defined populations with comprehensive benefits (with integrated inpatient, outpatient, and prescription drug services) that fee-for-service-based data sets could not generally render. It was also noted that consumers wanted research to help them reduce uncertainty about possible treatments, coordinate care, and aid in making appropriate decisions for care. ${ }^{9}$ Then and today, large employers want the best, most costeffective care for their employees and need better information to make coverage decisions.

The PBM industry finds itself positioned at a vital and influential junction of information flow from all major players in the health care industry. Some of the ties with players in the arena are purely on a financial basis; however, other, more interesting relationships have developed or may develop into something with a monumental influence for restructuring health services in the future. The simultaneous and complementary focus on outcomes research and evidence-based clinical decision making has highlighted the need for research to be carried out in real-world settings-not just relying on information from randomized clinical trial data gathered under artificially constructed settings. Although the latter historically generated a scientific basis for drug efficacy, the former "naturalistic" setting works best for the determination of effectiveness, or what the marketplace now demands as cost effectiveness. ${ }^{10}$

Extracting and modifying a summary question from the 1995 conference addressing research in the HMOs, one could pose a similar question for PBMs: Can the PBM industry and the research communities work together to create a climate in which decisions about health policy, organization, and practice can be, at least in part, grounded in scientific evidence about what really works, what does not work, what circumstances are necessary, what costs are involved, and which patients benefit?

\section{ISSUES FOR CONDUCTING HEALTH SERVICES RESEARCH}

Scientific research requires a serious, objective commitment to following the sequence of asking the research questions, designing a study to answer them, implementing appropriate methodologies, collecting data, analyzing and interpreting the results, and drawing conclusions. There has been no paucity of research questions. Pharmacists have been generating them over the years in efforts to improve pharmaceutical care, and the scientific literature can point the way toward more knowledge needed for pharmacotherapy advancement in practice. However, in the context of conducting health services research based on gathered data and information, the panel discussion in New Orleans dwelled on issues surrounding the availability and utility of assembled data.

Administrative databases capturing claims-based information share their history with the development and growth of HMOs. However, according to Darrell Newcomer, Director of Therapeutic Marketing for Healix Optimum Therapeutics, the information contained within these PBM databases is a "mishmash" of data elements with little standardization. What originally was collected by providers was billable utilization data under fee-for-service reimbursement; they gathered what they were told was necessary to get paid by insurers, who displayed little interest in the health status of the population. HMOs are paid prospectively, and no extensive effort has been made to transition their information systems toward aiding clinical decision making, partly because of the investment costs as well as the unavailability of advanced computer systems. The old-style HMO and preferred provider organization (PPO) databases are financially driven, primarily to monitor utilization in order to direct means to control costs. Even today, many HMOs have yet to emerge from this myopic vision of controlling costs purely through utilization control.

Control over the health information management (HIM) function rarely rests in the hands of the medical director. In fact, it is not uncommon for medical and pharmacy directors to be held hostage by the HMO database managers. Thus, MCOs usually observe patient encounters to minimize excessive utilization rather than to analyze clinical findings with the goal of improving patient health outcomes.

\section{DATA SOURCES}

Essentially, patient care information is captured entirely by three major components:

Continued on page 26 


\section{Continued from page 24}

$\Delta$ Inpatient utilization includes data on patient demographics, insurance details, billing for services offered and procedures performed (and their respective costs or charges), and final diagnoses in the hospital setting.

A Outpatient utilization includes data on patient demographics, insurance details, billing for services offered and procedures performed (and their respective costs or charges), and final diagnoses made in ambulatory care settings, such as physicians' offices, clinics, and home health agencies.

A Pharmacy utilization (often both inpatient and outpatient) includes information on drugs prescribed, the number of days of supply and dosage, the physician, the pharmacy, and associated insurance details.

Inpatient and outpatient information on diagnoses and procedures usually is gathered and reported based on the U.S. Health Care Financing Administration's Diagnosis-Related Group codes and the CPT-4 system, respectively. Most of the inpatient and outpatient data-hereafter referred to as medical data-must be manually entered into computer systems; scanning mechanisms still are not widespread. On the other hand, Otto Wolke, vice president of pharmacy, Geisinger Health Systems, pointed out that pharmacy data are semiautomated and usually require the pharmacist to enter only details such as the number of days' supply or modifications to the prescription. Because each insurer requires a different format, making queries of the database is often difficult. All three data sources have names and patient identifiers, but these may not be common throughout the provider organization, even for many MCOs and integrated health systems. All the sources probably contain financial fields or might be linked to the financial databases.

Most industry professionals will agree that the cost/reimbursement engine has driven the design and use of HIM systems. These systems originated to satisfy the insurance industry; thus, top provider managers have used them almost exclusively for financial control. As documentation of value for payment and quality assurance is demanded, clinical purposes will gain in importance and practitioners may have greater influence in the design of these systems. Newcomer believes that HEDIS (Health Plan Employer Data and Information Set) accreditation standards will push the industry in this direction.

\section{DATA COLLECTION}

In the past, pharmacy data were gathered separately from other patient care data. However, with increased realization of the folly of carving out this vital aspect of delivering care, a movement toward gathering information about the entire clinical experience is under way. The current PBM databases with information on millions of covered lives vary in the type and amount of information gathered as well as in the pace of planning for integration of data. As one can expect in a still-fledgling industry, PBMs have widely varying capabilities. There is, at present, no known standardization in the methods of data collection. With the recognized benefit of electronic medical record systems and the automation of the data gathered, the margin of human error involved in gathering and reporting clinical information can be expected to decline.

In addition to the human error (both intentional and unintentional) involved in data entry, there is a growing concern among insurers and MCOs about obtaining information from practitioners who are being paid under capitation. Wolke noted that capitation of providers is fast becoming the preferred means of payment and cost containment of MCOs. But getting timely and relevant clinical information from providers will remain problematic until advanced health information systems are successfully implemented, and used properly. ${ }^{11,12}$ Guidelines on the type of data to be gathered from each source await devel- opment, at least until the largest PBMs fashion their new integrated databases.

\section{DATA INTEGRATION}

Because medical and pharmacy data traditionally have been gathered separately, the dilemma facing researchers is the integration of the two data sets for a more complete picture. The complexities of such an integration are at times insurmountable. The information often is incomplete to begin with, and, in many cases, follow-up with patients is impossible. Patients may drop out of health plans; thus, long-term clinical follow-up does not take place and longitudinal therapeutic interventions are not tracked, documented, or analyzed thoroughly. Therefore, the implications of the care delivered are difficult to evaluate. As Tom Hughes, director of outcomes research at PCS Health Systems stated, "We're in an age where we are awash in data, yet starving for information."

\section{VALIDITY, REFRESH RATE, AND DIVERSITY OF DATA}

As information becomes more and more valued, questions about the validity of data gathered become more pronounced. Validity of data can be addressed only by standardization of the procedure used to gather information and with random audits comparing the data entered with the actual patient charts. As we move from "managing access to managing care," according to Hughes, it would be prudent to gather additional information on indirect economic costs, behavioral and lifestyle indicators, health status measures (such as quality of life), and, last but not least, productivity indicators, such as work loss and absenteeism.

The refresh rate of data refers to the frequency of updating within a specific database. Pharmacy data handled by most PBMs usually is updated in a matter of hours and days. However, medical information usually is updated every three months or every quarter, making it nearly impossible to intervene in the case of an identified problem. 
In light of such adversities, many studies are being conducted in-house with pharmacy data to identify drug interactions, appropriate age for use of specific drugs, appropriate indication of specific drugs, and so on. The growing influence of accreditation agencies, such as the National Committee on Quality Assurance and the Joint Commission on Accreditation of Health Care Organizations, and a demand for studies reflecting the quality of care provided have propelled development of clinical pathways and guidelines for clinical practice. The other influential players in goading MCOs to conduct evaluations of quality of care are employer groups and government agencies, who have begun to demand more information to establish the value of the care they are purchasing for their employees and beneficiaries.

\section{ROLE OF PBMs}

PBMs have emerged as the information managers in today's world of health care. They increasingly are urging MCOs to provide them with access to both medical and pharmacy data to manage total patient care information. PBMs currently sell themselves based on their capabilities to perform various activities, such as arranging disease state management programs, developing patient and provider educational programs, and performing retrospective and prospective drug utilization reviews. Yet, these services offered by PBMs are in response to customer demands. No known standards are established to compare the offerings, let alone the performance, of the 80-90 PBMs. A PBM digest publication with comparable data for each PBM would be a great benefit to the marketplace, but one has yet to be assembled. Thus, objectively differentiating the performance of various entities is difficult. PBMs have not been in the forefront on publishing results of studies they conducted, if such studies are being conducted at all. This might be partially due to an admitted limitation on the human resources to undertake detailed scientifically based studies, or due to the proprietary nature of such studies funded by individual manufacturers. Also, the reluctance of MCOs to share the medical information poses a problem; that reluctance is more or less driven by the economics of giving up expensive information, along with concern for the confidentiality of their enrolled patients. Purchasers appear to be content with increasing volumes of data assembled in PBM marketing proposals, which also never reach the public's eye.

The facilitation of health services research in these settings may be possible only through infrastructural investment in data management, along with the development of internal research proficiency through incorporating specialized talent and/or supporting individuals who can provide the methodological help. Newcomer noted that academic institutions and the pharmaceutical industry are two potential outside sources of intellectual expertise in conducting scientifically based studies. By collaborating with managed care residency programs, fellowships, and graduate programs, PBMs can get valuable assistance from their local institutions to carry out investigations. Such studies could be geared toward identifying means for maximizing pharmacotherapy access, improving quality, performing outcome studies based on self-reported or assessed outcome indicators (such as quality of life), identifying the actual cost of therapeutic interventions, and so on. Wolke reminded the audience that per-member/per-month drug costs still preoccupy the minds of most MCO managers.

\section{SUMMARY}

The progression of managed care as a mechanism for care delivery leads to a continuously increasing value expectation among purchasers. In turn, it is creating ripples throughout the health care organizational chain-most pointedly, the clear arrival of what Relman, former New England Journal of Medicine editor, identified as "the era of assessment and accountability."13

Outcomes research is here to stay. We must now discuss and debate how all health care parties can contribute to the overall advancement of delivery systems. We have in common the need to share learnings from research for the overall improvement of the health care system. Again, the staggering economic costs of drug misadventuring and its attendant human suffering require MCOs and PBMs to strive for disclosure of investigations that offer a complete clinical experience over time rather than mere episodes of care carved from prescription drug claims data or inpatient hospitalization bills. Evidence needs to be gathered from wider sources (i.e., naturalistic settings within managed care) rather than the limited, controlled environments of clinical trials. Newcomer insists that disclosure is important in terms of publication of results. Patient observational data such as quality of life, patient satisfaction, and other subjective measures should be given due consideration which, along with medical observations, include process and outcomes data, complications, and quality-adjusted life-years (QALYs) remaining.

In today's cost-conscious health system, data on resource use and related costs are deemed especially relevant. In addition to including data from a wide range of sources, the need to consider the perspective of different customers or clients is undeniable. Successful outcomes research requires an understanding of who the target audiences are and what the particular issues are that affect those specific audiences. Such issues may include demographics of the patient population, range of disease severity, data sources, and outcome and cost indicators used.

However, beyond customer and regulatory audiences lies the greater public's health. The uniqueness of the PBM industry lies in its intimacy with and unparalleled access to such data. The spotlight is on them for conducting health services research inside PBM 
quadrangles, and the potential rests with them for substantial contributions to improved performance for all pharmacotherapeutic interventions.

However, important issues must be deliberated and resolved to move forward in an organized and scientific manner. First, administrative databases have myriad limitations in providing useful information that could be generalized. ${ }^{10}$ This is purely by virtue of their creation solely to gather information for reimbursment purposes, with their legacy dating back to fee-for-service medicine. Second, the success in integrating information gathered on inpatient and outpatient bases depends on the completeness of such information in capturing the whole clinical experience longitudinally. Confidentiality and proprietary issues pose barriers in conducting research, especially in the dissemination of research results into the public domain. Only through discussion between the representatives of the interested parties-PBM firms, the research community, MCOs, the pharmaceutical industry, and other providers of care-can progress be assured with issues adequately resolved. Hughes sees the need for changing the incentive structure to alter organizational behavior of PBMs.

A tangible need exists to create a synergy between the various parties for facilitating research studies instrumental in providing cost-effective care, developing and implementing evidence-based guidelines and clinical pathways, creating disease state management programs, and more, for the ultimate purpose of improving overall public health. Our appeal is for PBMs to forge ahead in this critical exposition.

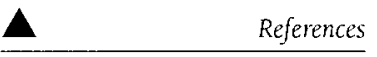

1. Manasse HR. Medication use in an imperfect world I: drug misadventuring as an issue of public policy. Am J Hosp Pharm 1989: 46: 929-944. 2. Johnson J, Bootman L. Drug-related morbidity and mortality: a cost-of-illness model. Arch Intern Med 1995(October); 155: 1949-56.3. Luft HS. Health maintenance organizations: dimensions of performance. New York: John Wiley, 1981

4. Anonymous. Evidence-based medicine working group, evidence-based medicine: a new approach to teaching the practice of medicine. JAMA 1992( November); 268: 2420-25.
5. Guadagnoli E, McNeil BJ. Outcomes research: hope for the future or the latest rage? Inquiry 1994(Spring); 31: 14-24

6. Sardinha C. AMCP speakers Reinhardt and Satcher paint bright future. J Managed Care Pharm 1997; 3(1): 11-16

7. Salmon JW, Goepel E, eds. International symposium on community participation and empowerment strategies in health promotion. Symposium papers presented at the ZIF Center for Interdisciplinary Studies. Bielefeld, Federal Republic of Germany, 1989.

8. Bernstein AB, Bernstein J, Shannon T, eds. Supplemental issue: building bridges between the $\mathrm{HMO}$ and health service research communities. Medical Care Research and Review 1996(March): 53(suppl)

9. Gaus CR. Bridging the gap: What can health services researchers and HMOs do for one another? Medical Care Research and Review 1996 (March); 53(suppl): \$12-\$17.

10. Gandhi S, Salmon JW, Kong SX, Zhao SX. (in review). Large patient databases and outcomes assessment: an overview of the issues and potential utility. J Managed Care Pharm 1998

11. Valenta AL, Wigger U. Q-methodology: definition and application in health care informatics. J Amer Informatics Assoc 1997(Nov).

12. Wigger, U. Medical accountability and information technology: medical students' and physicians' perspectives [unpublished dissertation]. University of Illinois at Chicago, Spring 1996. 13. Relman AS. Assessment and accountability: the third revolution in medical care. Engl J Med 1981; 319: 1220-22. 